\title{
Edge effects in chirped-pulse Fourier transform microwave spectra
}

\author{
G. Barratt Park ${ }^{\mathrm{a}}$, Robert W. Field ${ }^{\mathrm{a}}$ \\ ${ }^{a}$ Department of Chemistry, Massachusetts Institute of Technology, Cambridge, \\ Massachusetts 02139
}

\begin{abstract}
Recent applications of chirped-pulse Fourier transform microwave and millimeter wave spectroscopy have motivated the use of short (10-50 ns) chirped excitation pulses. In this regime, individual transitions within the chirped pulse bandwidth do not all, in effect, experience the same frequency sweep through resonance from far above to far below (or vice versa), and "edge effects" may dominate the relative intensities. We analyze this effect and provide simplifying expressions for the linear fast passage polarization response in the limit of long and short excitation pulses. In the long pulse limit, the polarization response converges to a rectangular function of frequency, and in the short pulse limit, the polarization response morphs into a form proportional to the window function of the Fourier-transform-limited excitation pulse.
\end{abstract}

\section{Introduction}

Chirped-pulse Fourier-transform microwave (CP-FTMW) spectroscopy, invented in Professor Brooks Pate's lab [1, 2], is rapidly becoming a mainstream technique for broadband (>10 GHz) high-resolution microwave and millimeterwave spectroscopy. Since its inception, CP-FTMW has also been a dynamicallyrelevant technique. Dian et al. [2] show that the spectral coalescence of the broadband rotational spectrum of a highly vibrationally-excited species can be

Email addresses: barratt@mit.edu or barratt.park@gmail.com (G. Barratt Park), rwfield@mit.edu (Robert W. Field)

Preprint submitted to Elsevier

March 13, 2015 
used to extract dynamical information about intramolecular vibrational energy redistribution (IVR) and chemical isomerization. Fine structure in the chirpedpulse spectrum of the prism isomer of the water hexamer enables the measurement of hydrogen-bond network rearrangement [3]. Prozument et al. [4] have used chirped-pulse millimeter wave spectroscopy to obtain meaningful product branching ratios and vibrational population distributions from a flash pyrolysis reactor. Recent work in the A. G. Suits lab $[5,6]$ has demonstrated the use of time-domain CP-FTMW measurements to measure the vibrationally-specific rate of appearance of unimolecular and bimolecular reaction products in a cold uniform flow.

Because of growing interest in CP-FTMW as a tool for kinetics and dynamics, there is an increasing necessity for reliable, quantitatively accurate measurement of relative intensity across broadband spectral regions. At the same time, several factors are driving researchers to use chirped excitation pulses of shorter duration. First, there has been a push to extend chirped-pulse spectroscopy to higher millimeter-wave frequencies, where Doppler dephasing times become short $[7,8,9]$. Next, the use of CP-FTMW by Abeysekera et al. [6] to study bimolecular reactions at pressures as high as $\sim 0.2$ mbar leads to a regime where the coherence time is shortened by pressure broadening. Indeed, the authors of Ref. 6 found that when using $1 \mu$ s long excitation pulses, dephasing phenomena had a significant effect on the relative intensities. As a result of dephasing during the excitation pulse, intensities of transitions that were excited early in the chirped pulse were weakened relative to those of transitions excited later in the pulse. The problem was mitigated by decreasing the pulse duration to $250 \mathrm{~ns}$, or by taking the frequency-domain average between spectra from "upchirp" and "down-chirp" excitation pulses. (See Figure 2 of Ref. 6.) Proposed experiments to probe pure-electronic transitions in Rydberg molecules rely on chirped pulse schemes for rapidly populating non-core-penetrating high- $l$ states before pre-dissociation occurs $[10,11]$. Finally, kinetic schemes involving timeresolved broadband microwave spectroscopy [6] motivate fast excitation pulses to achieve the best time-resolution for monitoring state-resolved reactant and 
product populations.

References 4 and 12 discuss the need for the chirped excitation pulse to be in the weak-field (fast-passage) limit rather than the strong-field (adiabatic rapid passage) limit in order to avoid saturation effects on the relative intensities. References 6 and 13 and others discuss the need for the duration of the excitation pulse to be short relative to the coherence decay time in order to achieve accurate relative intensities. However, most authors have ignored "edge effects" on relative intensities in chirped-pulse spectra. Therefore, we will discuss these effects briefly. These effects become pronounced as one uses shorter excitation pulses. It is important to point out that we do not derive any novel physics in this paper. Our results may all be obtained from analysis of the equations presented in Refs. 14 and 15 and reviewed in detail in Ref. 16.

\section{Polarization from a linearly chirped pulse in the weak coupling limit}

The polarization response $P=2\left(P_{r} \cos \omega t-P_{i} \sin \omega t\right)$ of a two-level system to a linearly swept excitation pulse of the form $E=2 \mathcal{E} \cos (\omega t-k z)$ is reported in the weak coupling limit by McGurk et al. $[15,14]$ and is also discussed in Ref. 13 and reviewed in detail in Ref. 16. If phenomenological dephasing terms are ignored, the optical Bloch equations for this situation is exactly solvable. The solution is given by

$$
\begin{array}{r}
P_{r}=\frac{\left|\mu_{a b}\right|^{2} \mathcal{E} \Delta N_{0}}{\hbar} \sqrt{\frac{\pi}{\alpha}}\left\{\sin \left(\frac{\left(\Delta \omega_{f}\right)^{2}}{2 \alpha}\right)\left[-C\left(\frac{\Delta \omega_{i}}{(\pi \alpha)^{1 / 2}}\right)+C\left(\frac{\Delta \omega_{f}}{(\pi \alpha)^{1 / 2}}\right)\right]\right. \\
\left.-\cos \left(\frac{\left(\Delta \omega_{f}\right)^{2}}{2 \alpha}\right)\left[-S\left(\frac{\Delta \omega_{i}}{(\pi \alpha)^{1 / 2}}\right)+S\left(\frac{\Delta \omega_{f}}{(\pi \alpha)^{1 / 2}}\right)\right]\right\} \\
P_{i}=-\frac{\left|\mu_{a b}\right|^{2} \mathcal{E} \Delta N_{0}}{\hbar} \sqrt{\frac{\pi}{\alpha}}\left\{\cos \left(\frac{\left(\Delta \omega_{f}\right)^{2}}{2 \alpha}\right)\left[-C\left(\frac{\Delta \omega_{i}}{(\pi \alpha)^{1 / 2}}\right)+C\left(\frac{\Delta \omega_{f}}{(\pi \alpha)^{1 / 2}}\right)\right]\right. \\
\left.+\sin \left(\frac{\left(\Delta \omega_{f}\right)^{2}}{2 \alpha}\right)\left[-S\left(\frac{\Delta \omega_{i}}{(\pi \alpha)^{1 / 2}}\right)+S\left(\frac{\Delta \omega_{f}}{(\pi \alpha)^{1 / 2}}\right)\right]\right\},
\end{array}
$$


where $C(a)$ and $S(a)$ are the Fresnel integrals,

$$
C(a)=\int_{0}^{a} \cos \left(\pi x^{2} / 2\right) \mathrm{d} x, \quad S(a)=\int_{0}^{a} \sin \left(\pi x^{2} / 2\right) \mathrm{d} x,
$$

$\mu_{a b}$ is the transition dipole moment, $\Delta N_{0}$ is the population difference of the twolevel system, $\mathcal{E}$ and $\alpha$ are the electric field strength and sweep rate of the driving pulse, respectively, and $\Delta \omega_{i}$ and $\Delta \omega_{f}$ are the initial and final values of the the detuning from resonance. We have simplified the expression derived by McGurk et al., noting that the Fresnel integrals are odd functions, $C(-a)=-C(a)$ and $S(-a)=-S(a)$, which allows us to remove the absolute values and the \pm choices.

The solutions (1-2) seem complicated, but the form is simplified by the assumptions

$$
\left|\Delta \omega_{i}\right| \gg(\pi \alpha)^{1 / 2}, \quad\left|\Delta \omega_{f}\right| \gg(\pi \alpha)^{1 / 2} .
$$

With this assumption, we can use the limiting behavior of the Fresnel integrals,

$$
\begin{array}{ll}
\lim _{a \rightarrow-\infty} C(a)=-0.5 & \lim _{a \rightarrow-\infty} S(a)=-0.5 \\
\lim _{a \rightarrow+\infty} C(a)=+0.5 & \lim _{a \rightarrow+\infty} S(a)=+0.5,
\end{array}
$$

We assume the case where $\Delta \omega$ is swept through resonance from negative to positive $\left(\Delta \omega_{i}<0, \Delta \omega_{f}>0\right.$, and $\left.\alpha>0\right)$. From assumption (3), we obtain

$$
\begin{aligned}
P_{r} & \approx \frac{\left|\mu_{a b}\right|^{2} \mathcal{E} \Delta N_{0}}{\hbar} \sqrt{\frac{\pi}{\alpha}}\left\{\sin \left(\frac{\left(\Delta \omega_{f}\right)^{2}}{2 \alpha}\right)-\cos \left(\frac{\left(\Delta \omega_{f}\right)^{2}}{2 \alpha}\right)\right\} \\
P_{i} & \approx-\frac{\left|\mu_{a b}\right|^{2} \mathcal{E} \Delta N_{0}}{\hbar} \sqrt{\frac{\pi}{\alpha}}\left\{\cos \left(\frac{\left(\Delta \omega_{f}\right)^{2}}{2 \alpha}\right)+\sin \left(\frac{\left(\Delta \omega_{f}\right)^{2}}{2 \alpha}\right)\right\} .
\end{aligned}
$$

In the case where $\Delta \omega$ is swept in the other direction, the phase of Eqs. (4$5)$ will change, but the magnitude will remain the same. Assumption (3) will hold for most chirped pulse experiments as long as the sweep rate is not too fast and the molecular resonance is not too close in frequency to the initial or final frequency of the linearly swept excitation pulse - i.e., if the transition is not too close to the high- or low-frequency edge of the spectrum. For a typical chirped pulse experiment, the excitation pulse sweeps through a bandwidth of 
$\sim 10 \mathrm{GHz}$ during a time interval of $\sim 1 \mu \mathrm{s}$, so $\alpha \approx 10^{16} \mathrm{~s}^{-2}$ and $(\pi \alpha)^{1 / 2} \approx 180$ MHz. Furthermore, in this case, the values of the square-bracketed terms in Eqs. (1-2) will be within $\sim 20 \%$ of unity for any transition not within $30 \mathrm{MHz}$ of the boundaries $\Delta \omega_{i}$ or $\Delta \omega_{f}$, so the approximation in Eqs. (4-5) will usually apply. With these typical conditions, the approximation will be invalid only for transitions located in the outer $0.3 \%$ wings of the frequency span of the applied pulse.

Equations (4-5) may be simplified even further to give the magnitude of the oscillating polarization. Recalling the definitions of $P_{r}$ and $P_{i}$, we see that the bracketed sin and cos terms in Eqs. (4-5) merely describe the rapid oscillation of the polarization in the rotating frame of the applied electromagnetic field. Because the polarization of the molecular resonance will oscillate at a frequency $\omega_{0}$ in the non-rotating frame, we may write

$$
P \approx \frac{2\left|\mu_{a b}\right|^{2} \mathcal{E} \Delta N_{0}}{\hbar} \sqrt{\frac{\pi}{\alpha}} \sin \left(\omega_{0} t+\phi\right)
$$

where $\phi$ is a phase that may be determined from Eqs. (4-5), and the prefactor, $2\left|\mu_{a b}\right|^{2} \mathcal{E} \Delta N_{0} / \hbar$ gives the magnitude of the oscillating polarization.

\section{Limiting behavior of the polarization response with respect to pulse duration}

For very short excitation pulses, assumption 3 is invalid. Therefore, it is useful to examine the limiting behavior of Eqs. (1-2) as $\alpha \rightarrow \infty$ and $\alpha \rightarrow 0$. The evolution of the polarization response from a $10 \mathrm{GHz}$ bandwidth excitation pulse is shown in Fig. 1. We define the nominal bandwidth, $\Delta \omega_{p}$, and duration, $T_{p}$, of the chirped excitation pulse such that

$$
\begin{aligned}
\Delta \omega_{p} & =\Delta \omega_{f}-\Delta \omega_{i} \\
T_{p} & =\frac{\Delta \omega_{p}}{\alpha} .
\end{aligned}
$$



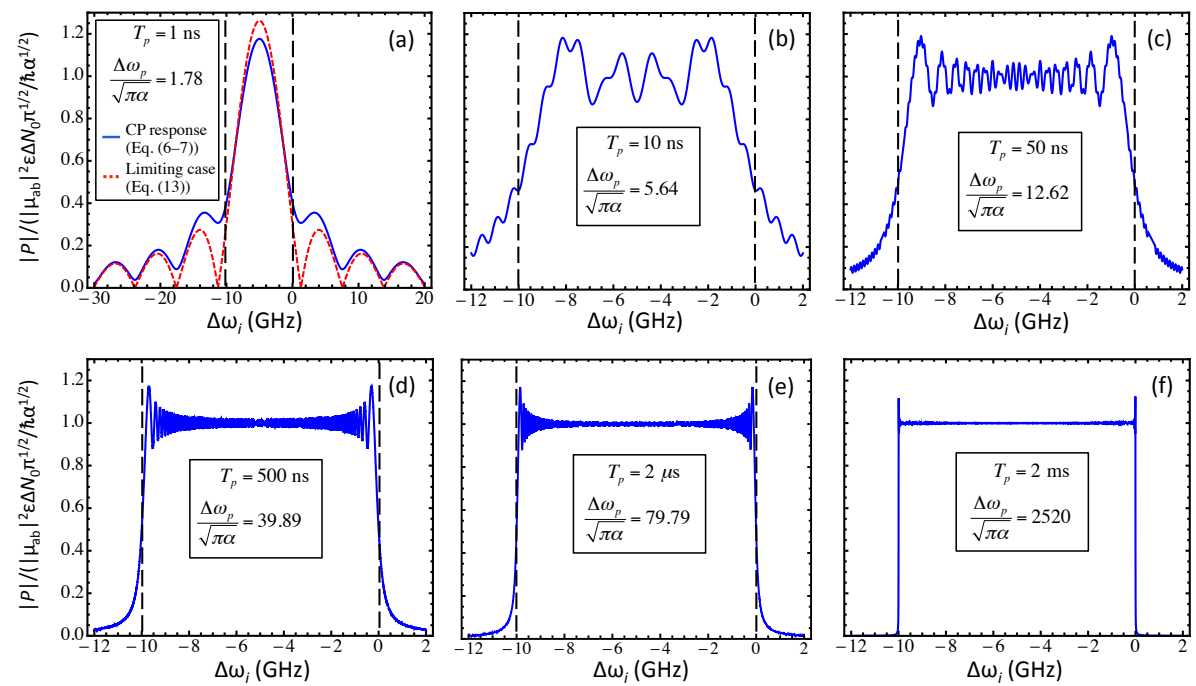

Figure 1: The magnitude of the polarization from Eqs. (1-2), normalized to the prefactor as indicated, is plotted against the initial detuning frequency $\Delta \omega_{i}$ for a $\Delta \omega_{p}=10 \mathrm{GHz}$ bandwidth chirped pulse as a function of pulse duration, $T_{p}$. In panel (a), the limiting sinc function behavior from Eq. 8 is plotted for comparison as a dotted curve, normalized to the same prefactor. As the pulse duration increases, the frequency dependence of the polarization response morphs from the window-function-limited behavior of Eq. 8 to the rectangular response function of Eq. 7 .

\subsection{Long pulse limit}

In the limit of very long pulse duration, $(\alpha \rightarrow 0)$, assumption (3) applies and the limiting behavior of the Fresnel integrals is relevant. Note that the signs of the Fresnel integrals in Eqs. (1-2) depend on the signs of $\Delta \omega_{i}$ and $\Delta \omega_{f}$. Assuming $\Delta \omega_{f}>\Delta \omega_{i}$, there are three cases:

$$
\lim _{\substack{\alpha \rightarrow 0 \\ \mu \mathcal{E} \rightarrow 0}}|P|= \begin{cases}0 & \Delta \omega_{f}<0 \text { and } \Delta \omega_{i}<0 \\ \frac{2\left|\mu_{a b}\right|^{2} \mathcal{E} \Delta N_{0}}{\hbar} \sqrt{\frac{\pi}{\alpha}} & \left(\Delta \omega_{i}<0 \text { and } \Delta \omega_{f}>0\right) \\ 0 & \text { or }\left(\Delta \omega_{i}>0 \text { and } \Delta \omega_{f}<0\right) \\ 0 & \Delta \omega_{f}>0 \text { and } \Delta \omega_{i}>0 .\end{cases}
$$

The first and third cases of Eq. (7) apply when the resonance is below or above the excitation pulse bandwidth and the second case applies when the resonance is within the excitation bandwidth. Thus, we see that in the $\alpha \rightarrow 0$ limit of an infinitely long excitation pulse, Eqs. (1-2) become a square wave excitation with 
a completely flat polarization response. (See Fig. 1(f).) Of course, Eqs. (1-2) also assume the weak field limit $\left(\mu \mathcal{E} / \hbar \ll \alpha^{1 / 2}\right.$, i.e. the Rabi frequency is slow compared with the sweep rate) and neglects dephasing. Thus, to obtain this result we have also implicitly assumed an infinitesimal $\mu \mathcal{E}$ and $1 / T_{2}$ coherence dephasing rate. Therefore, panel (f) of Fig. 1 is not intended to represent a physically realistic regime for most experiments - it is merely intended to illustrate the limiting behavior of Eqs. (1-2).

\subsection{Short pulse limit}

In the limit of very short pulse duration $(\alpha \rightarrow \infty)$, assumption (3) does not apply. However, in the limit where the bandwidth of the excitation pulse window function, $\mathcal{E}(t)$, becomes broader than the nominal chirp bandwidth, the polarization response becomes dominated by the Fourier transform of the window function. For a rectangular window function, it is possible to show that Eqs. (1-2) approach the limit

$$
\lim _{\alpha \rightarrow \infty}|P|=\frac{\left|\mu_{a b}\right|^{2} \mathcal{E} \Delta N_{0}}{\sqrt{2} \hbar} \frac{T_{p} \sin \left[\frac{1}{2}\left(\Delta \omega_{i}+\Delta \omega_{p} / 2\right) T_{p}\right]}{\frac{1}{2}\left(\Delta \omega_{i}+\Delta \omega_{p} / 2\right) T_{p}} .
$$

Eq. 8 is simply a sinc function centered at the middle of the chirped pulse bandwidth. Note that the $\sqrt{\alpha}$ scaling has disappeared and that the prefactor depends instead on the excitation pulse duration $T_{p}$. Thus, when the window function bandwidth becomes much broader than the chirp, the sample no longer behaves as if it is being excited by a chirped pulse. Instead, the polarization response morphs into what would be obtained from a rectangular excitation pulse with a carrier frequency at the center of the chirped pulse bandwidth. This limiting behavior is illustrated in Fig. 1(a). Note that in most chirpedpulse spectrometers, it is difficult to generate a 1 ns excitation pulse due to limitations of currently available microwave components. Therefore, the case illustrated in Fig. 1(a) is not intended to represent an experimentally realistic regime - it is merely shown to illustrate the limiting behavior of Eqs. (1-2).

In conclusion, there are three primary considerations that are necessary in order to ensure a flat polarization response across a broadband chirped-pulse 
spectrum:

$$
\begin{aligned}
T_{p} & \ll T_{2} \\
\left|\mu_{a b}\right| \mathcal{E} / \hbar & \ll \alpha^{1 / 2} \\
\left|\Delta \omega_{i}\right|,\left|\Delta \omega_{f}\right| & \gg(\pi \alpha)^{1 / 2}
\end{aligned}
$$

Condition (9a) ensures that the transition intensities will not be attenuated by dephasing of the coherence during the excitation pulse. As shown in Ref. 6 , dephasing effects can lead to anomalies in the relative intensities because transitions excited early in the excitation pulse will undergo more dephasing than transitions excited later in the excitation pulse. Condition (9b) ensures that the excitation pulse will be in the weak field limit so that saturation effects will be avoided and Eqs. (1-2) will be valid. Finally, condition (9c) ensures that the transition is not too close in frequency to either edge of the chirped-pulse bandwidth, where edge effects can dominate the relative intensities.

In the Bloch sphere picture, the two-level system is in the linear fast passage regime as long as two conditions apply. First, $\left|\Delta \omega_{i}\right|$ and $\left|\Delta \omega_{f}\right|$ must be much greater than $\left|\mu_{a b}\right| \mathcal{E} / \hbar$. That is, the initial detuning must be large enough to ensure that the driving vector, $\boldsymbol{\Omega}$, is, in effect, swept $180^{\circ}$ in the $u, w$ plane from the $+w$ to the $-w$ axis Bloch sphere axis. Secondly, $\alpha^{1 / 2}$ must be much greater than $\left|\mu_{a b}\right| \mathcal{E} / \hbar$ in order to avoid the rapid adiabatic passage (RAP) regime. When these two conditions are met, the Bloch vector, $\Theta$, initially precesses tightly around $\Omega$, but as the Rabi frequency decreases during the sweep toward resonance, $\boldsymbol{\Theta}$ becomes decoupled from $\boldsymbol{\Omega}$, avoiding RAP. We point out that these two conditions are insufficient to ensure a flat polarization response. In addition, it is necessary to have condition (9c) in order to avoid "edge effects." This places a lower bound on the amount of time in which the bandwidth $\Delta \omega_{p}$ can be rectangularly polarized. 


\section{Conclusions}

We have briefly described the impact of "edge effects" on the polarization response in linear fast passage experiments. In order to obtain a flat frequency response over the full bandwidth of the chirped excitation pulse, it is important for the initial and final detuning frequencies to be much greater than $\alpha^{1 / 2}$. This is because the linear fast passage regime relies on a Bloch vector, $\boldsymbol{\Theta}$, that follows the driving vector, $\boldsymbol{\Omega}$, adiabatically when it is far away from resonance, but becomes decoupled from the driving vector along the approach to resonance. If $\left|\omega_{i}\right|$ and $\left|\omega_{f}\right|$ are too small or $\alpha^{1 / 2}$ is too fast, the sample will not effectively experience a frequency sweep through resonance from far away, and the relative intensities will be affected by the window function of the chirped excitation pulse.

In the usual implementations of CP-FTMW, typically the bandwidth is not broader than $10-20 \mathrm{GHz}$ and the pulse duration is $1 \mu \mathrm{s}$ or longer, so that these effects are unimportant. However, new directions in chirped pulse microwave and millimeter wave spectroscopy have motivated the use of shorter excitation

pulses, where the polarization response loses its flatness. We have therefore analyzed the long-pulse and short-pulse limits of the linear fast passage response, and we have given the appropriate simplifying expressions for these extreme cases.

\section{Acknowledgements}

GBP would like to thank Arthur Suits for an insightful discussion that led to the current work. This paper is based upon work supported by the U.S. Department of Energy, Office of Science, Chemical Sciences Geosciences and Biosciences Division of the Basic Energy Sciences Office, under Award Number DE-FG0287ER13671.

[1] G. G. Brown, B. C. Dian, K. O. Douglass, S. M. Geyer, S. T. Shipman, B. H. Pate, A broadband Fourier transform microwave spectrometer based on microwave chirped pulse excitation, Rev. Sci. Instrum. 79 (2008) 053103. 
[2] B. C. Dian, G. G. Brown, K. O. Douglass, B. H. Pate, Measuring picosecond isomerization kinetics via broadband microwave spectroscopy, Science 320 (5878) (2008) 924-928.

[3] C. Pérez, M. T. Muckle, D. P. Zaleski, N. A. Seifert, B. Temelso, G. C. Shields, Z. Kisiel, B. H. Pate, Structures of cage, prism, and book isomers of water hexamer from broadband rotational spectroscopy, Science 336 (6083) (2012) 897-901.

[4] K. Prozument, G. Barratt Park, R. G. Shaver, A. K. Vasiliou, J. M. Oldham, D. E. David, J. S. Muenter, J. F. Stanton, A. G. Suits, G. Barney Ellison, R. W. Field, Chirped-pulse millimeter-wave spectroscopy for dynamics and kinetics studies of pyrolysis reactions, Phys. Chem. Chem. Phys. 16 (2014) 15739-15751.

[5] J. M. Oldham, C. Abeysekera, B. Joalland, L. N. Zack, K. Prozument, I. R. Sims, G. B. Park, R. W. Field, A. G. Suits, A chirped-pulse Fourier-transform microwave/pulsed uniform flow spectrometer. I. The lowtemperature flow system, J. Chem. Phys. 141 (15) (2014) 154202.

[6] C. Abeysekera, L. N. Zack, G. B. Park, B. Joalland, J. M. Oldham, K. Prozument, N. M. Ariyasingha, I. R. Sims, R. W. Field, A. G. Suits, A chirped-pulse Fourier-transform microwave/pulsed uniform flow spectrometer. II. Performance and applications for reaction dynamics, J. Chem. Phys. 141 (21) (2014) 214203.

[7] G. B. Park, A. H. Steeves, K. Kuyanov-Prozument, J. L. Neill, R. W. Field, Design and evaluation of a pulsed-jet chirped-pulse millimeter-wave spectrometer for the 70-102 GHz region, J. Chem. Phys. 135 (2) (2011) 24202.

[8] A. L. Steber, B. J. Harris, J. L. Neill, B. H. Pate, An arbitrary waveform generator based chirped pulse Fourier transform spectrometer operating from 260 to $295 \mathrm{GHz}$, J. Mol. Spectrosc. 280 (0) (2012) 3-10. 
[9] J. L. Neill, B. J. Harris, A. L. Steber, K. O. Douglass, D. F. Plusquellic, B. H. Pate, Segmented chirped-pulse Fourier transform submillimeter spectroscopy for broadband gas analysis, Opt. Express 21 (17) (2013) 19743-19749.

[10] K. Prozument, A. P. Colombo, Y. Zhou, G. B. Park, V. S. Petrović, S. L. Coy, R. W. Field, Chirped-pulse millimeter-wave spectroscopy of RydbergRydberg transitions, Phys. Rev. Lett. 107 (2011) 143001.

[11] A. P. Colombo, Y. Zhou, K. Prozument, S. L. Coy, R. W. Field, Chirpedpulse millimeter-wave spectroscopy: Spectrum, dynamics, and manipulation of rydberg-rydberg transitions, J. Chem. Phys. 138 (1) (2013) 014301.

[12] D. Schmitz, V. A. Shubert, T. Betz, M. Schnell, Multi-resonance effects within a single chirp in broadband rotational spectroscopy: The rapid adiabatic passage regime for benzonitrile, J. Mol. Spectrosc. 280 (0) (2012) $77-84$.

[13] V. V. Khodos, D. A. Ryndyk, V. L. Vaks, Fast-passage microwave molecular spectroscopy with frequency sweeping, The European Physical Journal: Applied Physics 25 (3) (2004) 203-208.

[14] J. C. McGurk, T. G. Schmalz, W. H. Flygare, Advances in Chemical Physics, Vol. 25, Academic, New York, 1974, Ch. 1, pp. 1-68.

[15] J. C. McGurk, T. G. Schmalz, W. H. Flygare, Fast passage in rotational spectroscopy: Theory and experiment, J. Chem. Phys. 60 (1974) 41814188.

[16] J.-U. Grabow, Fourier transform microwave spectroscopy measurement and instrumentation, in: M. Quack, F. Merkt (Eds.), Handbook of HighResolution Spectroscopy, Vol. 2, Wiley, 2011, pp. 723-799. 


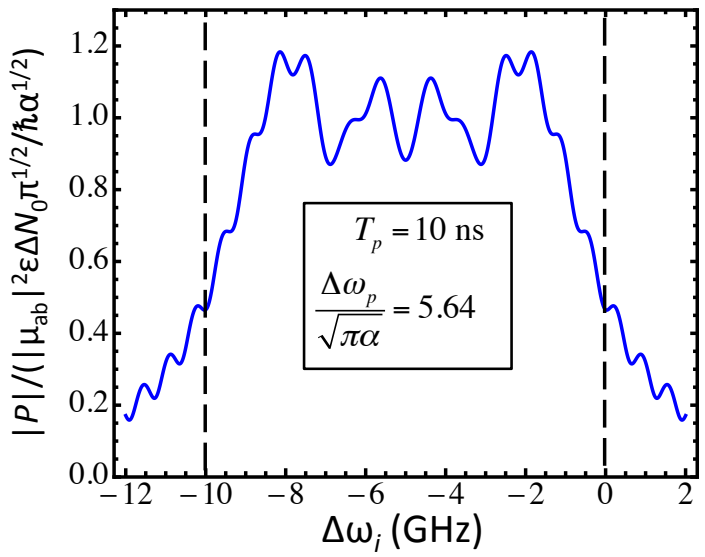

